\title{
Expansion to the Motion Sickness Susceptibility Questionnaire-Short Form: A Cross-Sectional Study
}

\author{
Emel Ugur ${ }^{1,2}$, Bahriye Ozlem Konukseven ${ }^{3}$, Murat Topdag ${ }^{4}$, \\ Mustafa Engin Cakmakci ${ }^{1,5}$, and Deniz Ozlem Topdag ${ }^{4}$ \\ ${ }^{1}$ Mehmet Ali Aydinlar University Vocational School of Health Sciences Audiometry, Istanbul, Turkey \\ 2Department of Audiology, Acibadem Altunizade Hospital, Istanbul, Turkey \\ ${ }^{3}$ Department of Audiology, Istanbul Aydin University Institute of Health Sciences, Istanbul, Turkey \\ ${ }^{4}$ Department of Otorhinolaryngology, Acibadem Altunizade Hospital, Istanbul, Turkey \\ ${ }^{5}$ Department of Otorhinolaryngology, Acibadem Bakirkoy Hospital, Istanbul, Turkey
}

Received October 6, 2021

Revised December 10, 2021

Accepted December 30, 2021

\section{Address for correspondence \\ Emel Ugur, Msc, Aud \\ Mehmet Ali Aydinlar University \\ Vocational School of Health Sciences \\ Audiometry, Kayisdagi Caddesi \\ No 32 Atasehir, Istanbul, Turkey \\ Tel +905336331934 \\ Fax +902166495370 \\ E-mail_emel.ugur@acibadem.com}

Background and Objectives: The primary objective of this study is to investigate the necessity of questioning virtual reality systems in the motion sickness susceptibility questionnaire (MSSQ)-short form. The secondary objective of this study is to determine the validity and reliability of the Turkish version of the MSSQ-short form, with proven validity and reliability. Subjects and Methods: In the questionnaire form, for which expert opinion was obtained to maintain linguistic equivalence, the virtual reality items were added to the questionnaire. The questionnaire was then administered to 297 individuals. The results were statistically analyzed with and without these virtual reality items for validity and reliability. Results: After the addition of the virtual reality items, the reliability of the questionnaire was found to be quite high (Cronbach's alpha $r=0.912$ ). The norm values between the original MSSQ-short form $(12.9 \pm 9.9)$ and the Turkish MSSQ-short form (13.8 \pm 12.9$)$ were found to be consistent. Conclusions: Motion sickness symptoms can occur not only during movement, but also with indirect stimulus. Our findings suggest that adding virtual reality items to the original form is important in long term practical applications. Our results show that the Turkish version of the original questionnaire is quite reliable. Submission of the MSSQ-short form in Turkish will be useful for documentation and will also encourage further research in this area.

J Audiol Otol 2022;26(2):76-82

Keywords: Motion sickness; Virtual reality; Questionnaire; Proprioception.

\section{Introduction}

One of the milestones in human history was developing means of transport by sea and maritime transport in commerce. Such a development eventually led to the rise of merchant shipping. The term "naus" from Greek nausea, which is generally associated with motion sickness (MS), is the sense of "sickness caused during a sea voyage" as defined by Hippocrates about 2,000 years ago [1-3].

Motion has been defined in all cultures as a critical stimulus

This is an Open Access article distributed under the terms of the Creative Commons Attribution Non-Commercial License (https://creativecommons.org/licenses/by-nc/4.0/) which permits unrestricted non-commercial use, distribution, and reproduction in any medium, provided the original work is properly cited. that causes MS [4]. Resulting in many concurrent symptoms, MS occurs not only during sea voyages but also on land, in the air, and even in space following the development and evolution of technology and means of transport. It was historically defined as MS as such symptoms are provoked by motion [5].

Research on the pathophysiology of MS is still ongoing. The vestibular apparatus has been included in the pathogenesis of MS since the beginning of the twentieth century. All species utilize the vestibular system as a generator to actively use the phase-locking mechanism between the vestibulo-ocular reflex and the vestibulospinal system for their basic needs, such as survival, nourishment, and shelter. MS is associated with susceptibility to inconsistent inputs as perceived from the vestibular, visual, and somatosensory systems and the in- 
ability to quickly adapt to such perceptive circumstances, i.e., sensory conflict (SC)/mismatch. This theory is one of the most fundamental theories on MS. Since patients with bilateral vestibular loss do not suffer from MS, it was established that the vestibular system induced MS [1,3,6].

Moreover, the vestibulo-cardiovascular reflex (VCR) was highly emphasized. The VCR is believed to occur due to the stimulation of otoliths with linear acceleration to induce a pressure response (increased blood pressure and cardiac output) as mediated by vestibulo-cardiovascular projections. Some studies suggest that specific symptoms of MS, such as nausea, tachycardia, perspiration, and discomfort, may result from the inappropriate activation of VCR based on motion. In addition, visual and vestibular systems and postural changes affect homeostatic equilibrium and the autonomic nervous system [7].

Questionnaires designed by Reason \& Brand were the first motion sickness susceptibility questionnaires (MSSQs) adopted as the earliest systematic measurement tools. These questionnaires were revised by Golding in 1998 (MSSQ-Long). As it contained 54 items and took a relatively long time to answer, Golding revised the questionnaire once more in 2006 to transform it into the MSSQ-short form (MSSQ-SF) containing 18 items. Since virtual reality (VR) systems were not on par with today's technology and, therefore, exposure was relatively less, they were excluded from the MSSQ-short form designed by Golding. In fact, in 2006, items referring to visual devices as cinema, video games, and VR sickness items were deliberately removed from the MSSQ during the development process because, at the time, visual devices as we know them today were not as common. Therefore, in the original study, the prevalence in the factor analysis was low and indicated no significant data in terms of variance [7].

The use of the MSSQ-SF has become the best practice to predict traditional motion sickness; however, the MSSQ-SF was not designed to predict visual induced motion sickness (VIMS) or cybersickness. There are various measurement tools in the literature that aim to measure VIMS and cybersickness [8,9].

Considering the awareness of the use of MSSQ-SF, it would be useful to add items related to VR and video game simulators to the scale. The primary purpose of this study is to investigate the necessity of adding the VR item to the questionnaire in terms of prevalence and variance.

Although the MSSQ-SF was translated into various languages and normalization efforts have been made, it has not been translated into Turkish, and its normalization process has not been undertaken yet. Therefore, there is no standardized questionnaire currently used in Turkey for the pre-diagnosis of MS.
MSSQ-SF will be applied to those who are living in Turkey and whose native language is Turkish. Therefore, the questionnaire will be adapted to Turkish, and its normalization will be provided. Thus, this questionnaire which has international validity and reliability, which is the secondary purpose of the study, will be introduced into Turkish. The adaptation of this questionnaire into Turkish would allow otorhinolaryngologists and audiologists, and neurologists to maintain a standard inventory for MS.

\section{Subjects and Methods}

The research was discussed in Acibadem Mehmet Ali Aydinlar University Acibadem Health Organizations Medical Research Ethics Committee ATADEK meeting on the date 09.01.2020, resolution 2020-1st and was approved with resolution 2020$01 / 48$. Written informed consent was obtained from the patients.

The MSSQ-SF has been translated into various languages for adaptation. For this study, first of all, the questionnaire was translated into Turkish; then, written expert opinion was obtained by interviewing three experts from the Audiology and Otorhinolaryngology departments who spoke both Turkish and English. These experts possess comprehensive knowledge about the etiology and symptoms of MS as they are involved in the follow-up, treatment, and rehabilitation of this patient population.

\section{Sample group}

The Turkish version of the MSSQ-SF, consisting of two parts and 20 items covering childhood and adulthood periods, was used. The questionnaire was completed by 297 (174 female/124 male) individuals between 18 and 45 (27.7 \pm 7.7$)$ years of age. In addition, volunteers comprising of university students, university staff, hospital staff, and the public were included in the study.

\section{Measurement tool}

The Turkish version of the MSSQ-SF, consisting of two parts and 18 items covering childhood and adulthood periods, was used. Considering technological development processes, VR item was added as simulation video games for the childhood period and VR gaming systems for the adulthood period. These items, which were added to the questionnaire for the requirement of its inclusion, were analyzed in terms of prevalence and variance. VR items were added to both parts of the questionnaire with the aim of learning about the effects of VR on MS. For validity and reliability analyses, it was advised that 180 individuals, which was calculated as ten times the number of 
reliable items $(10 \times 18)$, were involved in the study. Moreover, 1 item for each part (childhood and adulthood periods) of the questionnaire was added in order to investigate the provocative effects of VR on susceptibility to MS. Therefore, it was determined that the sample group size should consist of a minimum of 200 individuals.

\section{Data collection}

The questionnaire was administered face-to-face to the participants included in the sample group in person. First, participants were informed about the intended purpose of the questionnaire and how to evaluate the questionnaire. Then, the questionnaire was given to individuals who accepted to participate.

\section{Statistical analysis}

Data distribution was analyzed based on the Shapiro-Wilk test. The Mann-Whitney U test was used in the between-group variance of the data, which did not show normal distribution. Spearman's correlation coefficient was used for the evaluation of the correlation between the forms with and without the VR items, as well as for the analysis of total item correlations. Cronbach's alpha coefficient was used for the evaluation of reliability when the VR items were added to the current questionnaire. Exploratory factor analysis was performed based on equamax rotation, and principal component extraction methods during the analysis of the questionnaire construct when the item was added, and confirmatory factor analysis was conducted based on structural equation modeling (SEM) during the analysis of the construct validity. In the path diagram obtained as a result of confirmatory factor analysis, estimation values are provided. IBM SPSS Statistics 22.0 software (IBM Corp., Armonk, NY, USA) was used for statistical analyses, and LISREL 8.4 (Scientific Software International Inc., Lincolnwood, IL, USA) was used for SEM. All analytical results were reported with $\alpha=0.05$ significance and a $95 \%$ confidence interval.

\section{Results}

In the questionnaire form, for which expert opinion was obtained to maintain linguistic equivalence, the VR item was added as simulation video games for the childhood period and VR gaming systems for the adulthood period. Then the questionnaire was applied to 297 individuals.

\section{Norm}

An average total score of $13.8 \pm 12.9$ as obtained from the MSSQ-SF (excluding VR) indicated a positively skewed dis- tribution. An analysis of the descriptive statistical value of the MSSQ-SF with VR indicated a positively skewed distribution with an average total score of $15.1 \pm 13$.9. Based on an analysis of the variance between the sub-group mean values for the childhood and adulthood periods, the mean value for the childhood period was found to be higher with statistical significance than the mean value for the adulthood period $(p<0.001$; $\mathrm{U}=21299$; $\mathrm{Z}=-3.907$ ) [child: $8.23 \pm 7.59$ (median: 6 , min: 0, max: 27), adult: $5.54 \pm 6.06$ (median: 3.72 , min: 0, max: 27)]. When the scores for the childhood and adulthood periods were compared between the questionnaires with VR and without VR, the mean score for the childhood period was 6 (minmax: 0-27) without VR and 6.7 (min-max: 0-30) with VR, which indicated no statistically significant difference ( $p=0.589$, $\mathrm{U}=26153, \mathrm{Z}=-0.527)$. The mean score for the adulthood period was 3.75 (min-max: 0-27) without VR and 4 (min-max: $0-30)$ with VR, which indicated no statistically significant difference ( $p=0.384, \mathrm{U}=25663.50, \mathrm{Z}=-0.870)$. For the analysis of the reliability of total scores between the questionnaires with VR and without VR based on sex, statistically significant and highly positive reliability between the scores with and without VR was found for female $(\mathrm{r}=0.993 p<0.001)$. Statistically significant and highly positive conformity between the scores with and without VR was also found for male ( $r=0.994$ $p<0.001)$.

\section{Reliability}

The overall Cronbach's alpha value was found as considerably high as 0.935 based on the reliability analysis conducted without the addition of the simulation video games item. The correlation between part A and part B was found to be $\mathrm{r}=$ $0.612, p<0.001$ without the addition of the item, whereas the overall Cronbach's alpha value was found to be 0.912 after the addition of the item (VR). An analysis of the correlation between part A and part B indicated a moderately significant and positive correlation $(\mathrm{r}=0.623, p<0.001)$.

Although adding the VR item after the analysis of Cronbach's alpha values indicated a minor decrease, the reliability was found to be high. The relevant tables on the reliability analysis are as follows (Table 1).

Since there is a statistically significant and highly positive correlation $(\mathrm{r}=0.995, p<0.001)$ between the two questionnaires based on the correlation with and without the VR item, the retest reliability of the test ensures a reliability estimate of about 0.99 .

\section{Exploratory factor analysis (child \& adult)}

Since there was no item with a variance value below 0.50 based on the common variance results after conducting the 
Table 1. Motion experience, sickness frequency, and item correlations with the whole MSSQ-added VR item

\begin{tabular}{|c|c|c|c|c|c|c|c|c|}
\hline \multirow[t]{2}{*}{ Questionnaire Item } & \multicolumn{2}{|c|}{$\begin{array}{c}\text { Traveled or } \\
\text { experienced }(\%)\end{array}$} & \multicolumn{2}{|c|}{$\begin{array}{l}\text { Any sickness: } \\
\text { rarely or more (\%) }\end{array}$} & \multicolumn{2}{|c|}{$\begin{array}{c}\text { Sickness frequency } \\
\text { scale (mean) }\end{array}$} & \multicolumn{2}{|c|}{$\begin{array}{c}\text { Correlation with } \\
\text { VR (r) }\end{array}$} \\
\hline & Child & Adult & Child & Adult & Child & Adult & Child & Adult \\
\hline Car & 99.3 & 100 & 45.8 & 30.2 & 0.93 & 0.49 & 0.336 & 0.504 \\
\hline Bus & 99.0 & 100 & 57.6 & 43.4 & 1.27 & 0.82 & 0.292 & 0.421 \\
\hline Train & 74.4 & 89.2 & 22.6 & 17.9 & 0.57 & 0.30 & 0.349 & 0.524 \\
\hline Aircraft & 54.5 & 100 & 16.1 & 21.9 & 0.46 & 0.28 & 0.350 & 0.390 \\
\hline Small boat/rowboat & 68.0 & 100 & 28.2 & 29.3 & 0.76 & 0.42 & 0.394 & 0.338 \\
\hline Ship, ferry, etc. & 86.2 & 100 & 34.4 & 37.4 & 0.72 & 0.53 & 0.406 & 0.502 \\
\hline Swings & 98.3 & 100 & 32.0 & 28.6 & 0.60 & 0.45 & 0.323 & 0.416 \\
\hline Ferris wheel & 91.2 & 100 & 52.5 & 43.7 & 1.11 & 0.79 & 0.462 & 0.470 \\
\hline Roller coasters, wave swinger & 69.4 & 100 & 39.4 & 42.1 & 1.14 & 0.76 & 0.413 & 0.522 \\
\hline
\end{tabular}

The $p$-value for correlation coefficients is $p<0.001$, which indicates the $p$-value for Spearman's correlation analysis. MSSQ, motion sickness susceptibility questionnaire; VR, virtual reality

exploratory factor analysis on 20 items (10 for child and 10 for adult), as included in the questionnaire in order to determine the reliability and validity of the questionnaire, these 20 items (child and adult) were all included in the subsequent factor analysis.

Based on the results, the Kaiser-Meyer-Olkin value was 0.749 . The fact that $0.749>0.60$ and Bartlett's Test result was significant $(p<0.001)$ indicates that the questionnaire was suitable for factor analysis. Therefore, the results suggested high correlations between the items. The equamax rotation method was used to ensure that the factors matched the items with high correlation in the study. As a result of varimax rotation, four factors with an eigenvalue above one were found in the questionnaire. The eigenvalues of the 4 factors that were created are as follows respectively: Factor 1 eigenvalue $=8.465$, Factor 2 eigenvalue $=2.100$, Factor 3 eigenvalue $=1.721$, and Factor 4 eigenvalue $=1.546$. Collectively, these four factors account for $69.16 \%$ of the total variance. The factor weights and exploratory total variance percentage of the questionnaire as created based on the results of the exploratory factor analysis are provided in Table 2.

\section{Item-total score correlation}

The internal consistency of the final version of the MSSQshort form, as designed to measure the MS of the participants in the study Spearman's correlation coefficient, was used in the calculation of the total item correlation. As a result of the item-total score correlation analysis, the correlations were found to be positive and robust (Table 3 ).

\section{Structural equation modeling}

Following the factor analysis for the addition of the simulation video games item to the questionnaire, confirmatory factor analysis was conducted in order to examine the con- struct validity of the final version of the questionnaire. For this purpose, the model to be tested was created using $20 \mathrm{ob}-$ served variables and four latent variables (road, maritime, aviation, and entertainment). The analyses were performed using LISREL 8.4 software. The fit index values for this measurement model were found to be the following: degree of freedom $=164(p<0.001)$, comparative fit index $=0.95$, root mean square error of approximation $=0.075$, non normed fit index $=$ 0.95, and normed fit index $=0.92$. Based on the results, the goodness of fit index values for the fit criteria are presented in Table 4.

The fit index values as obtained from the confirmatory factor analysis indicate that the model fits the data. According to this result, no adjustment was carried out by linking together the error variances between the items. The measurement model and the results for the model are presented in the path diagram (Fig. 1).

\section{Discussion}

The original MSSQ-SF was administered to participants after they had been provoked under controlled laboratory conditions. In this study, however, the participants did not undergo any provocation method; they were simply asked to answer the questions in the questionnaire based on their previous experiences.

The original MSSQ-SF excluded the items of VR systems. The primary objective of this study is to examine whether the inclusion of these two items in the questionnaire alters the validity and reliability of evidence and whether such inclusion would contribute to the measurement. The conformity between the original MSSQ-short and the Turkish MSSQshort remained constant after the addition of these two items $(15.1 \pm 13.9)$. 
Table 2. Exploratory factor analysis details of the MSSQ

\begin{tabular}{|c|c|c|c|c|}
\hline \multirow{2}{*}{ Item } & \multicolumn{4}{|c|}{ Factor weights } \\
\hline & 1 (entertainment) & 2 (maritime) & 3 (road) & 4 (aviation) \\
\hline \multicolumn{5}{|l|}{ Child } \\
\hline Car & & & 0.803 & \\
\hline Bus & & & 0.859 & \\
\hline Train & & & 0.628 & \\
\hline Aircraft & & & & 0.803 \\
\hline Small boat/rowboat & & 0.768 & & \\
\hline Ship, ferry, etc. & & 0.695 & & \\
\hline Swings & 0.698 & & & \\
\hline Ferris wheel & 0.649 & & & \\
\hline Roller coasters, wave swinger & 0.633 & & & \\
\hline Simulation video games & 0.525 & & & \\
\hline \multicolumn{5}{|l|}{ Adult } \\
\hline Car & & & 0.787 & \\
\hline Bus & & & 0.820 & \\
\hline Train & & & 0.579 & \\
\hline Aircraft & & & & 0.791 \\
\hline Small boat/rowboat & & 0.775 & & \\
\hline Ship, ferry, etc. & & 0.700 & & \\
\hline Swings & 0.567 & & & \\
\hline Ferris wheel & 0.835 & & & \\
\hline Roller coasters, wave swinger & 0.744 & & & \\
\hline Simulation video (virtual reality) games & 0.548 & & & \\
\hline
\end{tabular}

Table 3. Item total correlation coefficients

\begin{tabular}{lcc}
\multicolumn{1}{c}{ Item } & $\begin{array}{c}\text { Total } \\
\text { item } \\
\text { correlation }\end{array}$ & $\begin{array}{c}\text { p- } \\
\text { value }\end{array}$ \\
\hline Child & & \\
Car & 0.703 & $<0.001$ \\
Bus & 0.767 & $<0.001$ \\
Train & 0.649 & $<0.001$ \\
Aircraft & 0.574 & $<0.001$ \\
Small boat/rowboat & 0.684 & $<0.001$ \\
Ship, ferry, etc. & 0.725 & $<0.001$ \\
Swings & 0.616 & $<0.001$ \\
Ferris wheel & 0.761 & $<0.001$ \\
Roller coasters, wave swinger & 0.782 & $<0.001$ \\
Simulation video games & 0.521 & $<0.001$ \\
Adult & & \\
Car & 0.624 & $<0.001$ \\
Bus & 0.648 & $<0.001$ \\
Train & 0.565 & $<0.001$ \\
Aircraft & 0.588 & $<0.001$ \\
Small boat/rowboat & 0.574 & $<0.001$ \\
Ship, ferry, etc. & 0.723 & $<0.001$ \\
Swings & 0.633 & $<0.001$ \\
Ferris wheel & 0.704 & $<0.001$ \\
Roller coasters, wave swinger & 0.701 & $<0.001$ \\
Simulation video (virtual reality) games & 0.590 & $<0.001$ \\
\hline
\end{tabular}

The $p$-value is the value for Spearman's correlation analysis
Table 4. Goodness of fit indices

\begin{tabular}{lll}
\hline \multicolumn{1}{c}{ Fit indices } & \multicolumn{1}{c}{ Fit indicator } & Result \\
\hline Chi-Square/SD & $\leq 4-5$ & 2.65 \\
RMSEA & $0 \leq \mathrm{RMSEA} \leq 0.08$ & 0.075 \\
$\mathrm{CFI}$ & $0.90 \leq \mathrm{CFI} \leq 1$ & 0.95 \\
$\mathrm{NNFI}$ & $0.90 \leq \mathrm{NNFI} \leq 1$ & 0.95 \\
$\mathrm{NFI}$ & $0.90 \leq \mathrm{NFI} \leq 1$ & 0.92 \\
\hline
\end{tabular}

SD, standard deviation; RMSEA, root mean square error of approximation; $\mathrm{CFl}$, comparative fit index; $\mathrm{NFI}$, normed fit index; NNFI, Non Normed Fit index

MS symptoms occur not only during movement, that is, direct stimuli, but can also be induced by indirect stimuli [2]. Mallinson and Longridge [2] emphasized that many pursuits or even optokinetic stimulation, such as watching the clouds in the sky, could trigger MS symptoms. As a matter of fact, mobile devices are used very actively today. For example, the visual stimulus constantly moves while playing games, typing, or reading a message. Since such technological stimuli require the continuous and active use of the optokinetic stimulation mechanism, they actually cause a visual-vestibular mismatch. As a result, mobile device users now use more vestibulo-autonomous connections and experience MS provocation even more through these stimuli $[10,11]$.

Frequent encounters with such stimuli help individuals improve their ability to adapt to SC [11]. Since individuals have

80 J Audiol Otol 2022;26(2):76-82 


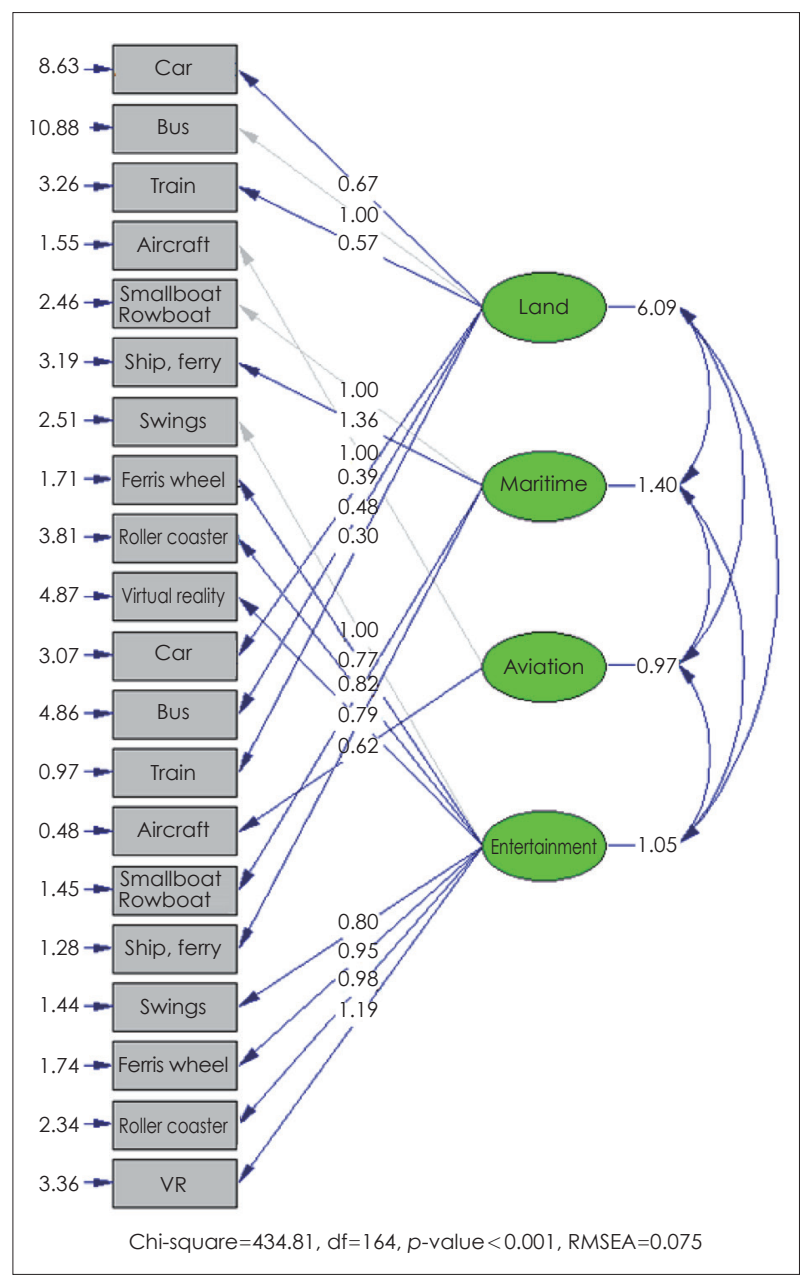

Fig. 1. The path diagram, measurement model and the results.

relatively less exposure to virtual environments, such as simulation video games and virtual reality systems, as they are not cost-effective, adaptation developed towards mobile devices may not be as effective in such environments. Therefore, it is crucial for these items to be included in the questionnaire for the diagnosis of MS. Internal consistency reliability $(r=0.912)$ as obtained from the questionnaire with added items was also consistent with the original version of the questionnaire. However, the statistical variance $(\mathrm{r}=0.912)$ from the questionnaire with added items was not significant despite this statistical consistency; in fact, also a minor decrease was observed. Also, there is a statistically significant and highly positive correlation $(r=0.995, p<0.001)$ between the two questionnaires based on the correlation with and without the VR item. In addition, the retest reliability of the test ensures a reliability estimate of about 0.99 . These findings suggest that adding such items to the original form and revising the form could be significant in the long term and practical applications considering the current technological equipment and media and the growth rate of technology.
For the analysis of the conformity of total scores between the questionnaires with VR and without VR based on gender, statistically significant and highly positive conformity between the scores with and without VR was found for both sexes. This finding indicates that VR applications are an effective factor for MS regardless of sex.

The secondary objective of this study is to prove the validity and reliability of the Turkish version of the MSSQ-short form, an internationally recognized MSSQ with proven validity and reliability. Hence, the aim is to make this questionnaire available in Turkish.

Motion sickness susceptibility is not observed in under two years. This is due to the fact that a baby is still in the process of developing spatial orientation skills. MS generally begins around the ages of 6 and 7 and reaches maximum levels during puberty due to hormonal changes. After puberty, adaptation gradually develops, or susceptible individuals develop protective or evasive behaviors. From the age of 50 onward, motion sickness susceptibility gradually decreases and then disappears $[1,6]$. Based on the results of the study, motion sickness susceptibility in children was found to be higher as compared to adults, which is consistent with both the literature and the original MSSQ-short.

Cronbach's alpha was used to calculate the internal consistency reliability of the Turkish MSSQ form. During the performance of the statistical calculations, part A, part B, and Total MSSQ scores were examined individually. Internal consistency reliability was calculated for both the original version and the version with the added items. The internal consistency of the Turkish version of the original questionnaire was the same as the original MSSQ-short form $(\mathrm{r}=0.935)$. This result suggests that the Turkish version of the original questionnaire is highly reliable.

The norm values between the original MSSQ-Short (12.9 \pm 9.9) and Turkish MSSQ $(13.8 \pm 12.9)$ were found to be consistent. The higher SD value based on the descriptive statistics as obtained from the Turkish MSSQ is probably due to the variance between the conditions of application of the questionnaires.

The use of the MSSQ-short form is not limited to the diagnosis of MS. It is also used in various pharmaceutical studies, migraine-associated vestibulopathy, migraine, and even Meniere's disease [12,13].

Even though its etiology is not well-established, it is a fact that the management of SC in postural disorders affecting the anatomy, such as scoliosis, is more challenging. For this reason, the MSSQ-short was also used in the literature to reveal MS susceptibility in scoliosis cases [14]. A study conducted by Uneri, et al. [15] is the only study in Turkey to sug- 
gest that scoliosis cases were susceptible to MS. However, the MSSQ-short form was not used in that study, and MS was diagnosed based on symptoms and medical history.

\section{Limitation of this study}

In this study, the participant group consisted of individuals between 18-45 ages. The questionnaire consists of two parts: childhood and adulthood. The questions of the adulthood part of the questionnaire aim to question the last ten years of experience. Considering the participants between the ages of 1820 , the most important limitation of this study is that the lower limit of the age range was determined as 18 years old. Although the inclusion of this age group in the study poses a risk in terms of practice, it was thought that their participation in the study would be important because they were familiar with VR systems. Therefore, while applying the scale form to these participants, they were asked to evaluate part B, especially considering the last eight years.

The number of participants between the ages of 18-20 and participating in the study is limited to only 26 people. However, these participants were not excluded from the study because it would not cause major changes in age-related statistics.

\section{Conclusion}

Making this MSSQ form available in Turkish will be useful for documentation and further research in the field.

\section{Acknowledgments}

We express our sincere gratitude to Aysegul Yabaci from the Biostatistics Department of the Bezmialem University for the technical support. This research did not receive any specific grant from funding agencies in the public, commercial, or not-for-profit sectors.

\section{Conflicts of interest}

The authors have no financial conflicts of interest.

\section{Author Contributions}

Conceptualization: Emel Ugur, Bahriye Ozlem Konukseven. Data curation: Emel Ugur. Investigation: all authors. Methodology: Emel Ugur, Bahriye Ozlem Konukseven. Project administration: Bahriye Ozlem Konukseven. Supervision: Bahriye Ozlem Konukseven. Validation: Bahriye Ozlem Konukseven, Murat Topdag, Mustafa Engin Cakmakci. Visualization: Emel Ugur. Writing — original draft: Emel Ugur. Writing — review \& editing: all authors. Approval of final manuscript: all authors.

\section{ORCID iDs}

Emel Ugur

Bahriye Ozlem Konukseven

Murat Topdag

Mustafa Engin Cakmakci

Deniz Ozlem Topdag

https://orcid.org/0000-0002-7908-5056

https://orcid.org/0000-0002-1409-0225

https://orcid.org/0000-0002-8683-1395

https://orcid.org/0000-0002-5940-5817

https://orcid.org/0000-0003-1797-1252

\section{REFERENCES}

1) Golding JF. Motion sickness susceptibility. Auton Neurosci 2006; 129:67-76.

2) Mallinson AI, Longridge NS. Motion sickness and vestibular hypersensitivity. J Otolaryngol 2002;31:381-5.

3) Oman CM, Cullen KE. Brainstem processing of vestibular sensory exafference: implications for motion sickness etiology. Exp Brain Res 2014;232:2483-92.

4) Huppert D, Benson J, Brandt T. A historical view of motion sicknessa plague at sea and on land, also with military impact. Front Neurol 2017;8:114.

5) Cheung B. Chapter 8-Seasickness: guidelines for all operators of marine vessels, marine helicopters and offshore oil installations. In: Survival at sea for mariners, aviators and search and rescue personnel (RTO-AG-HFM-152) (ed. Cheung RS, Reilly T, Gibbs P, Tipton MJ, Kozey J). Neuilly-sur-Seine: North Atlantic Treaty Organisation, Research and Technology Organisation;2008. p.1-24.

6) Paillard AC, Quarck G, Paolino F, Denise P, Paolino M, Golding JF, et al. Motion sickness susceptibility in healthy subjects and vestibular patients: effects of gender, age and trait-anxiety. J Vestib Res 2013; 23:203-9.

7) Golding JF. Predicting individual differences in motion sickness susceptibility by questionnaire. Pers Individ Differ 2006;41:237-48.

8) Kennedy RS, Lane NE, Berbaum KS, Lilienthal MG. Simulator sickness questionnaire: an enhanced method for quantifying simulator sickness. Int J Aviat Psychol 1993;3:203-20.

9) Kim HK, Park J, Choi Y, Choe M. Virtual reality sickness questionnaire (VRSQ): motion sickness measurement index in a virtual reality environment. Appl Ergon 2018;69:66-73.

10) Lackner JR. Motion sickness: more than nausea and vomiting. Exp Brain Res 2014;232:2493-510.

11) Van Ombergen A, Lubeck AJ, Van Rompaey V, Maes LK, Stins JF, Van de Heyning PH, et al. The effect of optokinetic stimulation on perceptual and postural symptoms in visual vestibular mismatch patients. PLoS One 2016;11:e0154528.

12) Golding JF, Patel M. Meniere's, migraine, and motion sickness. Acta Otolaryngol 2017;137:495-502.

13) Golding JF, Kadzere P, Gresty MA. Motion sickness susceptibility fluctuates through the menstrual cycle. Aviat Space Environ Med 2005;76:970-3.

14) Catanzariti JF, Guyot MA, Massot C, Khenioui H, Agnani O, Donzé C. Evaluation of motion sickness susceptibility by motion sickness susceptibility questionnaire in adolescents with idiopathic scoliosis: a case-control study. Eur Spine J 2016;25:438-43.

15) Uneri A, Polat S, Aydingoz O, Bursali A. Migraine vestibulopathy in three families with idiopathic scoliosis: a case series. Cases J 2009; 2:9367. 